\section{An e-Delphi Study on Software Virtualization in the Medical Diagnosis Process: The Experts' Perspective}

\section{Dr. Byrian L. Ramsey}

University of Phoenix

Submitted: 7 September 2020

Accepted: 12 November 2020

Published: 30 November 2020

Corresponding author:

Dr. Byrian L. Ramsey

DOI: $\underline{10.19044 / \text { esj.2020.v16n33p45 }}$

(c) Copyright 2020 Ramsey.

Distributed under Creative Commons

BY-NC-ND 4.0 OPEN ACCES

\section{Cite as:}

Ramsey. B, (2020). An e-Delphi Study on Software Virtualization in the Medical Diagnosis Process:

The Experts' Perspective. European Scientific

Journal, ESJ, 16 (33), 45.

https://doi.org/10.19044/esj.2020.v16n33p45

\begin{abstract}
The purpose of this qualitative e-Delphi study was to collect opinions and experiences from a group of expert panel members made up of physicians and virtualization experts. This e-Delphi research study provided information about the opinions and experiences from experts about the possible benefits for virtualization in medical facilities. The expert panel was comprised of 12 virtualization experts and physicians. Virtualization is a concept of technology where one physical hardware device, called a server, is configured with software that allows for multiple software servers to be installed or implemented. Virtualization may offer benefits to healthcare organizations including the ability to deliver patient data to physicians more quickly. Virtualization has demonstrated substantial benefits in many areas of information technology infrastructure in the medical informatics field. The expert panel members were chosen at random through existing professional relationships and previous employment. The expert panel members were provided three rounds of survey questions and were asked to complete all surveys in its entirety. The findings show that hospitals and medical facilities will benefit from virtualization and provide increased patient care by delivering faster data to the patient and physician. As leaders in hospitals and medical facilities continue to be well-informed, leaders will be better equipped to make more-informed decisions about virtualization and the positive relationship software virtualization has on patient care.
\end{abstract}

Subject: Health Technology 
European Scientific Journal, ESJ

November 2020 edition Vol.16, No.33
ISSN: 1857-7881 (Print) e - ISSN 1857-7431

Keywords: Virtualization, Health Information Systems, Hospital, Patient, Healthcare, Patient Relationship, Virtual, Technology, Information Technology 


\section{Introduction}

Receiving timely diagnoses from hospitals, health care providers, and physician practices [medical facilities] is a priority for patients. The increase in quality patient health care continues to be a concern of medical facilities. Patients desire a higher quality of care with precise diagnoses to sustain a healthier life. With the onset of technology, medical facilities continue to investigate solutions to increase the visibility of expedient quality services. Technology in health care continues to evolve and provides medical facilities new capabilities and functionalities for increasing the quality of care for patients. As technologies continue to evolve, new types of technologies have emerged that could assist in providing increased medical care to patients. As medical facilities continue to examine new technologies, some medical facilities are hesitant to implement new technologies due to risks, education, or budgeting costs and constraints (Daaleman \& Mueller, 2004; de Mul \& Berg, 2007; Parente \& McCullough, 2009; Purcarea, Petrescu, Gheorghe, \& Petrescu, 2011; Reiner, 2011; Takakuma, Shofer, Boedec, \& Reyes, 2003; White, 2008; Young, Mintz, Cohen, \& Chinman, 2004).

There are different types of technology evolving that may benefit medical facilities. One evolving technology is software virtualization. Software virtualization, also called virtualization, is a software increasing the capabilities of the medical facilities. Virtualization is a concept of technology where one physical hardware device, called a server, is configured with software that allows for multiple software servers to be installed or implemented (Microsoft, 2019; VMware, 2019). Two of the prevailing organizations that have developed virtualization are Microsoft ${ }^{\circledR}$ and VMware ${ }^{\circledR}$. The fundamental purposes of virtualization are to increase stability, reliability, expandability, and disaster recovery (Microsoft, 2019; VMware, 2019). When multiple software servers are built within a single hardware server, the hardware server is called a host server (Microsoft, 2019; VMware, 2019). In larger infrastructures, many hosts are implemented to carry software servers across multiple hosts to allow for increased performance, fault tolerance, and disaster recovery when one host goes offline. According to Loveland, Dow, LeFevre, Beyer, and Chan (2008), virtualization is software that emulates hardware. Virtualization does not depend on hardware to operate and operating systems do not require certain hardware to function. There may be benefits of using virtualization in medical facilities (Graschew et al., 2006; Kilman \& Forslund, 1997; Robb, 2008).

Health informatics is a rising field in health care. Health informatics continues to play an important role in medical facilities, such as hospitals and private practices. Traditionally, health care informatics provides information regarding the patient via paper or film. In the age of digital, health informatics has evolved to implement digital capabilities to handle patient medical 
information. The challenge of understanding software virtualization is a paradigm shift in knowledge with traditional managers and executives in medical facilities. Microsoft ${ }^{\circledR}$ and VMware ${ }^{\circledR}$ continue to push forward with new technologies in software virtualization to demonstrate to organizations the need for implementation (de Mul \& Berg, 2007; Parente \& McCullough, 2009; Purcarea et al., 2011; Reiner, 2011; Takakuma et al., 2003; White, 2008; Young et al., 2004).

Providing top quality patient care is a priority of medical facilities (de Mul \& Berg, 2007). Physicians and medical professionals look to technology to increase efficiency by delivering reliable and quality data at higher rates of speed (Young et al., 2004). With advancements in technology, medical facilities are delivering medical data quicker, more reliable, and more efficiently. Traditional methods of delivering medical data, such as computer hardware solutions and paper, may reduce the effectiveness for patients to receive timely, quality patient care. As such, medical facilities desire robust methods for delivering reliable and efficient medical data. Software virtualization has evolved providing multiple levels of redundancy, expandability, reliability, and sustainability (Microsoft, 2019; VMware, 2019).

Health care professionals and physicians may require a paradigm shift in knowledge to serve a higher quality of patient care (White, 2008). To be effective with providing timely feedback to patients, doctors require quick access to electronic patient data; however, elements exist influencing the timely access to patient data. One element influencing patient care is the timely delivery of patient data. According to Cantrill (2010), a growing issue is the delivery of patient data. Cantrill (2010) provided information showing how a simple procedure escalates out of control due to the lack of medical data not presented in a timely manner. Patients rely on the expertise of physicians and if physicians do not have timely access to medical data, patient care is impacted. According to Allgar and Neal (2005), the delay in medical treatment for six cancer patients proves to be evident in the outcome of the treatment of those patients. After surveying 65,192 patients, Allgar and Neal (2005) provided information to show that slower computer technologies affect the quality of the patient. The timely access to patient data is important to providing timely diagnoses.

Virtualization may offer benefits to health care organizations including the ability to deliver patient data to physicians more reliability and quicker. The general problem is that patient diagnoses are delayed to patients due to unreliable and underperforming server hardware. According to Cantrill (2010) and Graschew et al. (2006), health care facilities continue to be reluctant to implement virtualization due to the uncertainty of the technology. Organizations are fearful of placing several software servers on one hardware 
platform because of the "all eggs in one basket" theory. This theory describes the architecture of one hardware platform with several servers built on top of a single platform (Park \& Sharma, 2009). If the single platform goes offline or down, then all software virtual servers are offline affecting the delivery of patient data to physicians. Although Microsoft ${ }^{\circledR}$ and VMware ${ }^{\circledR}$ have technology built in that eliminates this theory from occurring, medical facilities continue to refrain from implementing software virtualization solutions (Microsoft, 2019; VMware, 2019).

The specific problem is that insufficient empirical evidence on experts' opinion exists on the relationship of software virtualization on the patient diagnosis process. Sufficient empirical evidence on experts' opinion is important for the benefit of patients and medical facilities. Without sufficient evidence on the relationship of software virtualization, patients may not receive proper health care and medical diagnoses. Patient care has been reduced due to hardware and non-standard practices for hospital and medical facilities infrastructures. As a result, patients have been receiving reduced medical care and delayed diagnoses due to unreliable data and the availability of data.

The purpose of this e-Delphi study was to collect opinions and experiences from a group of expert panel members made up of physicians and virtualization experts on the relationship of software virtualization on the patient diagnosis process. The expert panel answered three rounds of surveys online with SurveyMonkey®. Round one consisted of questions gathering information from the background of the expert panel and the panel's knowledge of virtualization, while Round two consisted of questions about the advantages and disadvantages of virtualization, and finally, Round three consisted of questions about the current and future state of virtualization. This research study collected opinions and experiences from a panel of experts in virtualization in medical facilities. The expert panel members were comprised of 12 virtualization experts and physicians.

This research study followed the same flow. First, the Delphi requirements were defined, which was the framework of the student and the type of research panel necessary for research. This research study identified the expert panel would consist of physicians and virtualization experts in the medical field. The second step of this study was to compose the number of expert panel members in this study. This study chose to represent 12 expert panel members. According to Loo (2002), the ideal study size for a Delphi study is approximately 20 participants. The population of expert panel members comprised of 12 medical informatics physicians and virtualization experts certified and their opinions and experiences in software virtualization in medical facilities. 
The third step of the theoretical framework was the gathering of the experts' opinion. The gathering of the expert opinions was cyclical to narrow the questions to the research question of this study. This research study consisted of three rounds to gather the opinions of the expert panel members. The expert panel answered three rounds of surveys online with SurveyMonkey®. Round one consisted of questions gathering information from the background of the expert panel and the panel's knowledge of virtualization, while Round two consisted of questions about the advantages and disadvantages of virtualization, and then finally Round three consisted of questions about the current and future state of virtualization. After three rounds of surveys, the final step of the theoretical framework was the statistical response. This study used NVivo ${ }^{\circledR}$ to analyze the pattern and themes emerging from the panel responses.

Porter (2008) created a tool that analyzes the competition of businesses and the external forces impacting the business. Porter's model contains five forces, such as supply-side economics of scale, demand-side benefits of scale, customer switching costs, capital requirements, and incumbency advantages independent of size (Porter, 2008, p. 27).

Porter's (2008) five forces are forces that influence business competition are similar to forces that can influence this research study. To ensure the framework was not influenced by outside forces, the study's structure was controlled by ensuring the physicians and software virtualization experts contained the appropriate certifications, knowledge, and information to align with the study's purpose and research.

Significant of the study for research. This research study is significant to the field of study for research of medical informatics in medical facilities to continue increasing patient care quality by increasing the delivery performance of patient diagnoses. Medical informatics continues to grow and evolve in different types of computer technologies. Two types of medical informatics data are cardiology and radiology data. Despite the two types of medical informatics data, each type is substantially different in how much data traverses the network and the type of data. Medical facilities continue to require challenging methods for delivering large amounts of data to physicians to make timely, informed diagnoses. This research is significant in the study for research in that the research builds upon previous research in virtual technology. As technology continues to change for medical facilities, continued research is necessary to study the relationship of software virtualization and other technologies on the impact of patient care and diagnoses.

Significance of the study for theory. This research study is significant for theory in that the study builds upon the theories of virtualization. This 
research study expands upon the existing theory of virtualization and the relationship of software virtualization on the patient diagnoses process.

Significance of the study for leadership. This research study is significant for leadership by providing research information to medical facility leadership and administration to assist in making informed decisions about delivering timely patient care with virtual technologies. Medical facilities continue to strive to make fiscally conscience decisions about proving higher patient care (Reiner, 2011; Schimke, 2009). One aspect of virtualization that reduces medical facilities operational and capital expenses is that virtualization reduces the necessity for hardware (Microsoft, 2019; Spink et al., 2016; VMware, 2019). In reducing the expenses in information technology, the medical facilities can use those resources for other means to increase patient quality or medical facility functionality.

The qualitative research method was appropriate for this research study because the information regarding the relationship of software virtualization in the medical diagnosis process was not numerical data but experiences and opinions from a panel of experts via online surveys. The expert panel member responded to three rounds of surveys to conform to a consensus.

\section{Research Question}

The following section provides the research question that guided this qualitative e-Delphi research study. The overarching research question in this study was:

What advantages and disadvantages did physicians experience with using virtualization during the patient diagnosis process?

\section{Theoretical Framework}

Porter (2008) created a tool that analyzes the competition of businesses and the forces that influence the business. Porter's model contains five forces, such as supply-side economics of scale, demand-side benefits of scale, customer switching costs, capital requirements, and incumbency advantages independent of size (Porter, 2008, p. 27).

Porter's (2008) five forces are forces that influence business competition are similar to forces that can influence this research study. To ensure the framework was not influenced by outside forces, the study's structure was controlled by ensuring the physicians and software virtualization experts contained the appropriate certifications, knowledge, and information to align with the study's purpose and research.

\section{Literature Review}

The history of virtualization dates back to the 1960s with the work of Melinda Varian (Ameen \& Hamo, 2013; Daniels, 2009; Gribbin, 1989; 
Miseviciene, Ambraziene, Tuminauskas, \& Pazereckas, 2012; Munro, 2001; Van Vleck, 2013). Melinda Varian played an essential role with the development and discovery of virtual machines for a Compatible TimeSharing System (CTSS) on the IBM OS/360 Model 67 (Ameen \& Hamo, 2013; Gribbin, 1989; Miseviciene et al., 2012; Munro, 2001; Van Vleck, 2013). Varian's research of virtualization provided the Massachusetts Institute of Technology (MIT) programming team the opportunity to expand further on Varian's idea to create a similar time-sharing computer or machine that provided more robust computing than the foundation of the system (Ameen \& Hamo, 2013; Daniels, 2009; Munro, 2001). In 1973, Srodowa and Bates demonstrated the process and steps for creating efficient virtual machines in relation to utilizing a new theory of hypervisor (Ameen \& Hamo, 2013; Daniels, 2009).

Evolving out of the work by Srodowa and Bates, a hypervisor is the software layer between the host operating system and the virtual machines on the server (Cervone, 2010; Daniels, 2009). The hypervisor is the layer that controls the communication between the hardware and the virtual machines to prevent direct access to the hardware (Ameen \& Hamo, 2013, p. 66). The hypervisor plays an important role in virtualization due to the barrier between that host's hardware and the virtual machines. MIT programmers discovered that by using the hypervisor this separates the virtual machines and allows the virtual machines to run independently from each other (Cervone, 2010; Daniels, 2009; Munro, 2001; Van Vleck, 2013). The independence of the virtual machines is necessary to separate the different memory spaces; thus allowing the virtual machines to be independent of each other and the host system (Cervone, 2010; Daniels, 2009; Munro, 2001; Van Vleck, 2013).

Before the 1980s, virtualization played a role within the mainframe computing systems; however, through the 1980s and 1990s, distributed architectures emerged with a more client-server based system and computing (Daniels, 2009). As a result, virtualization expanded into stand-alone servers and computing systems expanding the capability of the back-end systems while providing functionality for specific purposes, such as e-mail, databases, and applications (Ameen \& Hamo, 2013; Daniels, 2009; Gribbin, 1989; Van Vleck, 2013).

Since the 1990s, virtualization has expanded into larger organizations to reduce operational costs in data centers (Ameen \& Hamo, 2013). According to Ameen and Hamo (2013), "technological advancements in hardware and software make virtual machines stable, affordable, and offer tremendous value, given the right implementation” (p. 66). As virtualization continues to expand into organizations, new and prevailing capabilities in this technology have emerged and evolved. There are three primary types of virtual machines, such as software virtual machines, hardware virtual machines, and virtual 
operating system (OS) containers (Ameen \& Hamo, 2013; Daniels, 2009; Munro, 2001).

\section{Software Virtualization}

Virtualization is a phenomenon increasing the capabilities of the medical informatics field. According to Loveland et al. (2008), virtualization is software that emulates hardware. The virtualization software does not depend on hardware to operate and operating systems do not require certain hardware. Virtualization is relatively new to technology evolving since the early 1960's era (Ameen, \& Hamo, 2013; Daniels, 2009; Gribbin, 1989; Miseviciene et al., 2012; Munro, 2001; Van Vleck, 2013). Software virtualization benefits health informatics in several ways. Despite the benefits, organizations hesitate to implement software virtualization. To start effectively with the implementation, organizations must undergo a paradigm shift in technology knowledge. Organizations are fearful of placing several servers on one platform because of the "all eggs in one basket" theory. This theory or fear describes the architecture of one hardware platform with several servers building on top of a single platform (Park \& Sharma, 2009). Software virtualization provides a more efficient means for presenting server architectures and provides for a better foundation in providing quicker patient data to physicians. Virtualization continues to provide valuable benefits for information systems and technologies, such as lower hardware costs - reduces capital spending, increases server resources efficiency, increases uptime - fail over time between servers nodes is less than hardware, reduces the amount of power or electricity used in a data center, reduces the number of servers in the data center, faster server provisioning or imaging, better disaster recovery capabilities, more expandable, extends application life for older applications less dependent upon hardware, and the ability to isolate applications within a virtual environment (Armstrong et al., 2005; VMware, 2019).

e-Delphi research. The qualitative e-Delphi research study fits best for this research because a panel of experts answered open-ended, online questionnaires. This qualitative e-Delphi research study provides information from a panel of experts investigating if there are benefits for virtualization in medical facilities. The expert panel was comprised of 12 software virtualization engineers and physicians certified and experienced in the virtualization software technology in medical facilities.

\section{Research Methodology}

The process of collecting data through electronic measures are according to the Delphi Method Operations Flowchart (Hsueh, 2012, p. 2821). Hsueh (2012) developed the steps for a Delphi method to demonstrate the controlled nature of the study. "The Delphi method is composed of the 
following steps: (1) select experts; (2) obtain initial assessment factors from previous studies; (3) design and distribute questionnaires; (4) recover and modify questionnaires; (5) if assessment factors do not reach a consensus, return to Step 4; and (6) obtain the criteria required for this study" (Hsueh, 2012, p. 2821).

Table 1

Round 1 Survey Questions

\begin{tabular}{|c|c|}
\hline Number & Question \\
\hline 1 & What is your occupational role? \\
\hline 2 & $\begin{array}{c}\text { If physician, on average, how much time do you spend on each patient } \\
\text { during the diagnosis phase of interaction? }\end{array}$ \\
\hline 3 & If physician, on average how many patients do you diagnose per day? \\
\hline 4 & $\begin{array}{l}\text { If virtual expert, does your facility use virtualization? If so, what type (i.e. } \\
\left.\text { Microsoft }{ }^{\circledR} \text { or VMware }{ }^{\circledR}\right) \text { ? }\end{array}$ \\
\hline 5 & $\begin{array}{c}\text { If virtual expert, does the software used for medical diagnoses run in } \\
\text { virtualization? }\end{array}$ \\
\hline 6 & Do you know what virtualization is in technology? \\
\hline 7 & Describe an example of virtualization in technology. \\
\hline 8 & If physician, describe your patient diagnosis process. \\
\hline 9 & Does virtualization have any relationship on the patient diagnosis process? \\
\hline 10 & $\begin{array}{c}\text { Explain why or why not virtualization is or is not related to the patient } \\
\text { diagnosis process. }\end{array}$ \\
\hline 11 & What are the benefits of virtualization? \\
\hline 12 & $\begin{array}{l}\text { How might the benefits of virtualization benefit the patient diagnosis } \\
\text { process? }\end{array}$ \\
\hline
\end{tabular}

Round 2. Round 2 consisted of 3 questions (see Table 3). The purpose of round 2 was to gain information from the experience of the expert panel members through their knowledge of virtualization. Round 2 was designed to have both open-ended and closed questions. The open-ended questions were designed to continue discovering the advantages and disadvantages of virtualization with the expert panel respective organizations. The following were the questions in Round 2.

The purpose of question 1 was to gain information on the background of the panel member's role in the medical facility. This perspective was important to gain insight on which question the panel member should be answering during the study round. Questions 2 and 3 were questioned due to not gaining a consensus during Round 1 . Questions 2 was questioned to gain further insight and background from the expert panel members on the experienced disadvantages of software virtualization. In Round 1, question 11 (see Table 2) questioned the advantages of software virtualization; however, the data analysis showed that a consensus was not gained; therefore, asking the adverse provided additional information on the use of software virtualization. Question 3 gained additional insight into the relationship with software virtualization and the patient diagnosis process. The reason question 
3 was questioned a second time was due to the lack of a consensus and additional information and insight were required to form a consensus.

Table 1

Round 2 Survey Questions

\begin{tabular}{cc}
\hline Number & Question \\
\hline 1 & What is your occupational role? \\
3 & What are the disadvantages of using virtualization? \\
& How do you believe virtualization can impact the patient encounter \\
process?
\end{tabular}

Round 3. Round 3 consisted of 2 questions (see Table 4). The purpose of round 3 was to gain information on the future of virtualization. The questions were designed to understand how leadership can gain experience from the experiences of the expert panel. This research was designed to assist medical facilities in making informed decisions about using virtualization and the advantages and disadvantages of virtualization. The expert panel members were to provide background on their experiences and how leadership benefits from those experiences to make informed decisions. The following are the questions in Round 3.

Round 3, questions 1 and 2 were questioned to continue working towards a consensus. Question 1 gained information from the background of the literature review and the reasons from experiences why executive leadership has implemented software virtualization in the expert panel members' respective medical facilities. Question 1 was an important question to gain information from the panel's perspective on the relationship of software virtualization and the patient diagnosis process for gaining executive support. Question 2 was questioned to gain additional background on the future of software virtualization. The perspective from the expert panel on the future of software virtualization showed that software virtualization has a future in the medical sciences.

Table 2

Round 3 Survey Questions

\begin{tabular}{cc}
\hline Number & Question \\
\hline 1 & $\begin{array}{l}\text { If you are pitching an opportunity to your CEO, what information would } \\
\text { you present to remove the issues around having all "eggs in one basket" } \\
\text { with virtualization? }\end{array}$ \\
2 & Where do you believe virtualization is going over the next 5-10 years? \\
\hline
\end{tabular}

The three rounds were created to gain from the experiences of the expert panel members. The rounds were designed to continue to gather information to form to a consensus of the answers to the survey questions. 


\section{Analysis Software and Data Collection Portal}

NVivo ${ }^{\circledR} 12$ software is a qualitative research software developed by QSR International (QSR International, 2018). NVivo® software assists in the compilation of information and research data. NVivo ${ }^{\circledR}$ analyzes data by finding trends and emerging themes in survey responses. NVivo ${ }^{\circledR}$ is comprised of different analytical methods. This research study used cluster analysis within $\mathrm{NVivo}{ }^{\circledR}$. Cluster analysis is a technique used to explore patterns in data collection (QSR International, 2018). The analysis occurs by examining the data from the questionnaires and compiling a grouping or cluster of common terms or language in the questionnaire answers. NVivo ${ }^{\circledR}$ 12 was used in this research to analyze the cluster patterns of the expert panel responses. SurveyMonkey ${ }^{\circledR}$ is a web-based instrument for the collection of survey responses through online questionnaires. SurveyMonkey ${ }^{\circledR}$ was used in this research to collect the expert panel responses for three rounds of surveys. SurveyMonkey ${ }^{\circledR}$ also contains the ability to analyze survey responses via the cluster method. SurveyMonkey ${ }^{\circledR}$ contains different analysis methods; however, similarly to $\mathrm{NVivo}{ }^{\circledR}$, the cluster method was used in this research study.

\section{Population, Sampling, and Geographic Location}

The expert panel members were either virtualization experts or physicians in medical facilities across the United States. To participate in the study, the expert panel members have to be certified as software virtualization experts or a certified medical physician. The expert panel members either work, practice, or have experiences with the patient diagnosis process with virtualization in medical facilities across the United States. The sampling of participants occurred by random selection through previous business and personal contacts with the panel members. As each member agreed to perform on the research panel, the snowball effect also became prevalent for additional members. According to Egan and Estrada (2013), the snowball sampling effect is "designed to recruit recognized experts from the pool of acquaintances of other recognized experts" (p. 304). As the expert panel was solicited, it became prevalent that some of the expert panel members knew of other potential participants.

\section{Field Test}

The field test performed in this study utilized SurveyMonkey ${ }^{\circledR}$ and three software virtualization experts to test the questionnaire method and trustworthiness of the questioning process. First, the three software virtualization experts were questioned on the areas that could affect receiving appropriate information from the expert panel. After careful examination, the three experts determined there were no risks in the SurveyMonkey ${ }^{\circledR}$ 
questionnaire method. SurveyMonkey ${ }^{\circledR}$ contains internal controls to ensure questions were controlled and anonymous, according to settings setup during the survey creation process (SurveyMonkey, 2018).

Analysis and Results

\section{E-Delphi Rounds}

Round 1. The Round 1 questionnaire became available to participants on August 22, 2018, and ended on August 29, 2018. The expert panel members were emailed the SurveyMonkey ${ }^{\circledR}$ link to begin the questionnaire. The email was sent to all 12 expert panel members in such a way to ensure anonymity from the other panel members. 11 of the expert panel members elected to agree with the informed consent and complete the questionnaire. The Round 1 questionnaire consisted of eight open-ended questions and 4 multiple choice questions. Table 5 is the table of survey questions for Round 1 .

The purpose of Round 1 was to understand the background of the expert panel member and their experiences with virtualization in medical facilities. Question 1 was the informed consent where 11 of the 12 (92\%) participants elected to agree and move forward with the questionnaire. Question 2 defined the occupational role of the participant. Questions 3 through 6 collected data on the current knowledge of virtualization and what type of virtualization is used in the participant's medical facility. The data analysis shows that out of the 11 expert panel members, eight facilities utilize VMware ${ }^{\circledR}$ while three facilities use Microsoft ${ }^{\circledR}$ virtualization.

Question 7 of the survey questioned the expert panel member's background in virtualization. The data analysis shows that eight of the 11 (72\%) expert panel members understood virtualization technology. As technology changes, so do the ability to support and administrate the technology.

Question 7 responses were key indicators for Round 1 and the amount of information required for expert panel members to understand further the purpose of the research and the questions in the research questionnaire. Expert panel members were included in research due to their background and experience with virtualization; therefore, Question 7 is a key indicator to ensure the expert panel members are legitimate in knowledge and experience with virtualization.

Question 8 was for the physicians in the study to answer. The questions asked the physicians to provide details of their patient diagnosis process. Two physicians participating in the research study. One physician described the patient diagnosis process as a cycle. The physician described the process as an intellectual collection of data and then interpreting the data into a diagnosis. The data collection was a mental collection. The second physician described the diagnosis process as a data interpretation method. The second physician 
provided additional information stating that at times, the data was not arriving fast enough for the physician to make a quick diagnosis. The second physician provided additional details that substantiated the research that when data does not arrive to physicians quickly, the delay could cause a reduction in patient care. The second physician provided key information and with Question 11, additional information emerged. Question 8 received $100 \%$ participation from the physicians.

Question 9 was asked of both the physicians and virtual experts. Question 9 asked if virtualization has any relationship on the patient diagnosis process. Out of the 11 participants, nine of the participants provided answers to Question 9 (82\%). The purpose of this question was to gain information on the server infrastructure, if known, from the respondents. Question 10 was an open-ended question asking how virtualization is related to the patient diagnosis process. Nine of the 11 participants answered Question 10 (82\%). Question 11 of Round 1 was a key indicator of the study. Question 11 questioned the expert panel member how software virtualization was related to the patient diagnosis process. Question 11 was an essential indicator of the research study. Nine of the 11 participants answered question 11 (82\%). The final question of Round 1 was question 12, which asked the participants how the benefits of virtualization would benefit the patient diagnosis process. 10 and of the 11 participants answered question 12 (91\%). Round 1 provided intuitive experiences of the physicians and virtual experts. Unfortunately, a consensus did not conform; therefore, Round 2 was created to continue questioning the panel members about the benefits of virtualization.

Round 2. The Round 2 questionnaire became available to participants on September 30, 2018, and ended on October 7, 2018. Round 2 questionnaire was provided to the expert panel members via email for those who indicated they wanted to proceed with Round 2.11 of the 12 (92\%) original expert panel members indicated to proceed to Round 2. At the end of Round 2, eight of the 11 (73\%) participants completed the survey questions completely. Three of the 11 participants completed the informed consent to proceed; however, did not complete all survey questions in Round 2. Table 6 is the table of survey questions for Round 2.

Round 2 consisted of the informed consent to proceed and three openended questions. Question 1 was the informed consent where 11 of the 12 (92\%) participants agreed to move forward with the Round 2 questionnaire. The purpose of three Round 2 questions was to dive deeper into the knowledge of virtualization of the expert panel members. Question 2 gained information from the expert panel member of the disadvantages of using virtualization while the final question collected information on the impact of virtualization on the patient encounter process. In Round 1, Question 12 asked the expert panel member the advantages of virtualization; while Round 2, Question 3 
requested information on the disadvantages of virtualization. The purpose of asking contrasting questions in two different rounds was to gain further insight from the expert panel member on their experience and background of virtualization. Question 4 continued the discussion on the patient diagnosis process. Question 4 continued receiving the experiences of the panel members and how their experiences related to virtualization and the patient diagnosis process.

Round 3. The Round 3 questionnaire became available to participants on October 15, 2018, and ended on October 22, 2018. Round 3 consisted of three questions. Round 3 questionnaire was provided to the expert panel members via email for those who indicated they wanted to proceed with Round 3. Question 1 was the informed consent question. 11 of the 12 (92\%) original expert panel members indicated to proceed to Round 3. The purpose of the two questions was to gain information from the expert panel members regarding where virtualization will be going in 5-10 years and the type of information required to convince executive leadership to implement virtualization. Although $92 \%$ of the participants indicated to proceed with Round 3; six out of 11 (55\%) expert panel members completed Round 3. The loss of 5 expert panel members may be attributed to the amount of time required to participate in the study, personal or job commitments, the loss of interest in participating in the study, or the length of rounds for the study. No one indicated via email or phone call they were not continuing the study. Table 7 is the table of survey questions for Round 3.

Six participants answered both questions of Round 3. Although Rounds 2 and 3 had reduced expert panel members who participated in the study than Round 1 , the response rate was $55 \%$ for those who participated in the study. According to Yehuda (1999), the acceptance rate for responses depends on the study and the number of participants. For this study, the acceptable participation rate was 50\% which was achieved in each round of surveys.

\section{Results}

From the results of the data analysis emerged three primary themes from the expert panel members. After three rounds of interpretation, a consensus was formed and the following results occurred.

Theme 1: Increased performance with software virtualization. Theme 1 emerged from the responses multiple times. Expert panel members responded that software virtualization processes data for efficiently and quickly than traditional hardware platforms. Each of the expert panel members was uniquely identified via a lettering and numbering system, for example, P1 was Participant 1, P2 was Participant 2, etc. P1 stated, "higher resource availability and system HA [high availability] leads to higher up-times and better performance across clinical applications" P2 stated, "Having a virtual 
environment allows for use of routing technology like Akamai to route requests in the quickest way possible providing speed.” P4 stated, "virtualization can increase availability and performance for the virtualized service." P7 stated, "faster and more reliable patient care as technology becomes more and more a part of our system used to provide care.”P10 stated, "the ability to add resources to a virtualized instance running the patient diagnosis software in order to improve performance and the ability to migrate virtualized instances between physical hosts to limit the amount of downtime for the patient diagnosis application(s)." And finally, P11 stated, "Speed: Get results faster than running from a physical machine." The consensus and analysis resulted that software virtualization does increase system performance and the ability to deliver data faster to the physician for quicker medical diagnoses.

Theme 2: Increased data reliability with software virtualization. The increase in data reliability emerged as the second theme from the expert panel responses. The consensus showed that software virtualization increases data reliability, meaning the data will arrive more reliably with software virtualization than with traditional hardware infrastructures. Data reliability was also linked to higher availability of data. P4 stated, "high availability offers conservation of resources, shared physical resources, disaster recovery/business continuance offerings, faster reboot time for virtual machines, and faster deployment via cloning and template deployment operations.” P2 stated, "may help minimize downtime and be easier to support.” P7 stated, “creating a highly available and high performing system that supports clinicians benefits the patient. Saving money through virtualization can create funds for medical staff. Virtualization can also increase [the] mobility of information, allowing clinicians to more easily meet patient needs. That can include scenarios like a doctor's ability to visit the patient in any room, or other location, with quick access to the same software. Virtualization also benefits [the] analytical processes used to determine how effective clinical care is for patients. That information leads to company/hospital/clinical care improvements." P9 stated, "benefits include up time due to high availability, lower impact from hardware changes, faster restoration from backup, ease of management and visibility due to management software. In many cases, virtualization may save money.” And finally, P11 stated, "virtualization allows a business to utilize physical servers at a much more efficient capacity level, as guest operating systems are able to take advantage of idle Computer Processing Unit (CPU) cycles and unused Random Accessed Memory (RAM) on the host. This allows the business to save on physical footprint. It also affords the business much more mobility, as the virtual servers can be built at a pace much quicker than traditional physical server builds, that must be ordered, delivered, racked, and provisioned.” The 
consensus and analysis resulted that software virtualization does increase the reliability and availability through increased uptime for physicians to give more reliable patient diagnoses.

Theme 3: Reduction in cost with software virtualization. The theme for the reduction in cost emerged from the expert panel responses. The results show that implementing software virtualization will decrease the costs of the server infrastructure. P3 stated "it [software virtualization] allows the provider the ability to provide more patient-facing services at a lower cost as they are able to run more of those applications/services on less hardware or in multiple locations (on-premise vs cloud). It also allows them to provide higher availability for those services as they are able to implement redundancy at a lower cost." P4 stated, "the high availability offerings of virtualization can provide greater uptime and availability of critical healthcare systems much easier and cheaper than using physical servers." And finally, P5 stated "reduce costs by reducing the need for physical servers which in turn reduces licensing needs, energy and data center needs. It also allows ease of creating new servers and environments on the fly." The consensus and analysis resulted that software virtualization will reduce the operational costs for medical facilities.

\section{Conclusions and Recommendations}

Virtualization is the conceptual concept of technology that builds multiple servers onto one hardware platform (Microsoft, 2019; VMware, 2019). Virtualization may offer benefits to health care organizations including the ability to deliver patient data to physicians more quickly. The virtualization literature review validated that virtualization is an emerging technology that can affect the patient diagnosis process. The findings of this research study using expert panel members in virtualization aligned with the literature review for virtualization. The expert panel members concluded Round 3 that virtualization can positively affect the patient diagnosis process. The literature review indicated there were many benefits to virtualization and throughout the analysis, the benefits emerged to demonstrate that virtualization can positively impact the patient diagnosis process. The use of virtualization in the patient diagnosis process has been a concern of many medical facilities (Conti, 2001; Harbin \& Baum, 2014; Marco, 2004; Shapiro, 1993). A challenge for medical facilities is the ongoing costs to maintain computer technologies to provide quick access to patient data.

Theme 1: Increased performance with software virtualization. According to Loveland et al. (2008), virtualization is software that emulates hardware. Virtualization does not depend on hardware to operate and operating systems do not require certain hardware to function. As such, software virtualization processes data more quickly and has the ability to process big data more efficiently. As a result of the analysis, the findings 
concluded that virtualization positively affects the patient diagnosis process. The data analysis shows that patients can receive faster results with the use of virtualization than traditional hardware methods.

The hypervisor plays an important role in virtualization due to the barrier between that host's hardware and the virtual machines. MIT programmers discovered that by using the hypervisor the virtual machines are separated and allows the virtual machines to run independently from each other. The independence of the virtual machines is necessary to separate the different memory spaces; thus allowing the virtual machines to be independent of each other and the host system (Cervone, 2010; Daniels, 2009; Munro, 2001; Van Vleck, 2013). With the use of the hypervisor in software virtualization, performance increased due to software virtualization being able to process data more quickly and bigger amounts of data processed more efficiently.

Medical informatics organizations state that virtualization increases efficiency and dependability within the medical industry (Fujifilm, 2019). Microsoft ${ }^{\circledR}$ and VMware ${ }^{\circledR}$ recognize a growing trend in system architectures, especially the medical field, to decrease the footprint in data centers by decreasing the number of physical servers in the data center (Microsoft, 2019; VMware, 2019). As big data continues to evolve and require additional computing power, hardware manufacturers continue to build computer systems with larger processing speeds, faster memory processing, and increased video capabilities (Yaffe, 2019). Due to the development methods and how the data is processed, software virtualization does increase the performance of medical data.

Theme 2: Increased data reliability with software virtualization. Organizations are fearful of placing several servers on one platform because of the "all eggs in one basket" theory. This theory or fear describes the architecture of one hardware platform with several servers building on top of a single platform (Park \& Sharma, 2009). Software virtualization implementations with more than one host increase the reliability of data through continual uptime. The results from the consensus and analysis show that software virtualization provides more reliable data.

The fundamental purpose of virtualization is to increase stability, reliability, expandability, and disaster recovery (Microsoft, 2019; VMware, 2019). When multiple software servers are built within a single hardware server, the hardware server is called a host server (Microsoft, 2019; VMware, 2019). In larger infrastructures, many hosts are implemented to carry software servers across multiple hosts to allow for increased performance, fault tolerance, and disaster recovery when one host goes offline. The results from the analysis show that software virtualization increases data reliability through increased uptime. 
Theme 3: Reduction in cost with software virtualization. According to Bernstein et al. (2007), there are five constants of information technology that continue to drive success within medical facilities. Those five constants are "budget, supportive leadership, project management, implementation, and end user involvement" (p. 17). Despite these constants, medical facilities continue to slow down on integration and the effectiveness of information technology (Bernstein et al., 2007). With the implementation of software virtualization, medical facilities will reduce operating expenses in the information technology department.

One aspect of virtualization that reduces medical facilities operational and capital expenses is that virtualization reduces the necessity for hardware (Microsoft, 2019; Spink et al., 2016; VMware, 2019). In reducing the expenses in information technology, the medical facilities can use those resources for other means to increase patient quality or medical facility functionality. With the reduction in the quantity of server hardware, the information technology footprint is reduced. The results and consensus show that software virtualization decreases the cost of ownership for medical organizations.

As medical facilities continue to examine new technologies, some medical facilities are hesitant to implement new technologies due to risks, education, or budgeting costs and constraints (Daaleman \& Mueller, 2004; de Mul \& Berg, 2007; Parente \& McCullough, 2009; Purcarea et al., 2011; Reiner, 2011; Takakuma et al., 2003; White, 2008; Young et al., 2004). The results of this research study show that operational expenses are reduced; therefore, medical facilities will reduce budget costs.

\section{Contribution to Research}

This research study contributed to research by exploring the advantages and disadvantages of virtualization in the medical informatics field to assist in increasing the care of patients. The data analysis provided themes that showed that virtualization positively affected the patient diagnosis process; therefore, patients may receive more reliable information through virtualization than hardware.

The research study contributed to the knowledge of software virtualization and the relationship on the patient diagnosis process. Through this research, the opinions and experiences of the expert panel contributed to the further advancement in software virtualization in medical facilities.

\section{Contribution to Theory}

This research study contributed to the theory of virtualization by building upon the expandability and reliability of virtualization with respect to patient data and diagnosis. The theory that virtualization was a viable solution for medical facilities becomes more prevalent in the research of this 
study. The data analysis provided insight that virtualization does positively affects the patient diagnosis process; therefore, medical facilities would benefit from the implementation of virtualization.

\section{Contribution to Leadership}

This qualitative e-Delphi research study contributed to leadership by providing research information to medical facility leadership to assist in making informed decisions about delivering timely patient care. Medical facilities continue to strive to make fiscally conscientious decisions about proving higher patient care (Reiner, 2011; Schimke, 2009). One aspect of virtualization that reduces medical facilities operational and capital expenses is that virtualization reduces the necessity for hardware (Microsoft, 2019; VMware, 2019). In reducing the expenses in information technology, the medical facilities can use those resources for other means to increase patient quality or medical facility functionality.

The findings of this research study show significant analysis to provide hospital leadership that virtualization can improve patient care through timely diagnoses. The benefits for virtualization include high availability, increased reliability, and increased expandability, while lowering the cost of ownership to the organization. The expert panel members affirm that medical facilities would benefit from virtualization; however, the complexity of administration is greater.

\section{Strengths of the Study}

This research study demonstrated several strengths throughout the research. First, this study gained additional information on how data is gathered for the patient diagnosis process. Within the literature review, the study found information that although physicians were provided patient data, the process in which the data is obtained was different depending on the specialty of the physician. The second strength of the study was the use of an expert panel. The expert panel had direct experience with virtualization and patient diagnosis within a medical facility. The expert panel members' experiences contributed to the research

\section{Limitations of the Study}

This qualitative e-Delphi research study contains limitations. The first limitation is the classification of the expert panel of experts. The panel of experts is designated as experts per this research study's criteria. However, other criteria may exist that could prevent some panel members from being experts in certain aspects of the medical field. This limitation is controlled by designing the research panel around industry standards in the medical informatics field in cardiology and radiology. 
The number of expert panel members started out strong with 11 members; however, toward the end of Round 3, there were fewer number of expert panel members. The reduction in expert panel members may be attributed to the amount of time to participate in the study, personal or job commitments, the loss of interest in participating in the study, or the length of rounds for the study.

Another limitation that emerged during the analysis of the data was the length of the responses. Most of the expert panel members provided shorter answers than expanding on the question to provide deeper insight into the situation. Due to not providing lengthy responses to the open-ended questions, analyzing the themes were more challenging and some were incomplete. This limits the amount of information to analyze and could potentially affect the emerging conclusions from the data.

\section{Recommendation for Future Research}

This research study was limited in scope; however, there are recommendations for future research that emerged. The first recommendation for future research would be within other fields, such as financial and manufacturing. Financial and manufacturing may benefit from virtualization because these fields require quick, reliable data for fast decisions. Financial and manufacturing fields also require the processing of large amounts of data. AI may assist in the processing of large amounts of data.

The second recommendation for future research would be to implement software virtualization in medical facilities. The expert panel consensus confirms that software virtualization positively increases the care patients receive through reliable and more efficient data. Implementing software virtualization in medical facilities would increase patient care.

The next recommendation for future research is to research the relationship of software virtualization in expert systems. Expert systems will continue to evolve; however, with a relationship with software virtualization, expert systems may be able to process increased amounts of data under extreme processing speeds. Software virtualization was an initial step towards more efficient expert systems and the management of big data.

The final recommendation for future research is studying the relationship of software virtualization on data mining. Data mining manages large amounts of data and virtualization may assist in faster processing and increased capabilities for data mining experts. As more data requirements emerge, systems will need to consistently increase computing power and efficiency. 


\section{References:}

1. Allgar, V. L., \& Neal, R. D. (2005). Delays in the diagnosis of six cancers: Analysis of data from the National Survey of NHS Patients: Cancer. British Journal of Cancer, 92(11), 1959-1970. doi:10.1038/sj.bjc.6602587

2. Ameen, R. Y., \& Hamo, A. Y. (2013). Survey of server virtualization. International Journal of Computer Science and Information Security, 11(3), 65-74.

3. Armstrong, W. J., Arndt, R. L., Boutcher, D. C., Kovacs, R. G., Larson, D., Lucke, K. A., Nayar, N., \& Swanberg, R. W. (2005). Advanced virtualization capabilities of POWER5 systems. IBM Journal of Research and Development, 49(4.5), 523-532. doi:10.1147/rd.494.0523

4. Cantrill, S. (2010). Computers in patient care: The promise and the challenge. Communications of the ACM, 53(9), 42-47. doi:10.1145/1810891.1810907

5. Cervone, H. F. (2010). An overview of virtual and cloud computing. OCLC Systems and Services, 26(3), 162-165. doi:10.1108/10650751011073607

6. Conti, F. (2001). Claude Bernard: Primer of the second biomedical revolution. Nature Reviews. Molecular Cell Biology, 2(9), 703-8. doi:10.1038/35089594

7. Daniels, J. (2009). Server virtualization architecture and implementation. Crossroads, 16(1), 8-12. doi:10.1145/1618588.1618592

8. de Mul, M. M., \& Berg, M. (2007). Completeness of medical records in emergency trauma care and an IT-based strategy for improvement. Medical Informatics \& The Internet In Medicine, 32(2), 157-167. doi:10.1080/09670260701231284

9. Egan, A., \& Estrada, V. (2013). Socio-economic indicators for forest restoration projects. Ecological Restoration, 31(3), 302-316. doi:10.3368/er.31.3.302

10. Fujifilm Medical Systems. (2019). Retrieved from http://fujifilmusa.com/products/medical/index.html.

11. Graschew, G., Theo A., Roelofs, T. A., Rakowsky, S., Schlag, P. M., Heinzlreiter, P., Kranzlmuller, D., \& Volkert, D. (2006). New trends in the virtualization of hospitals - Tools for global e-health. Medical and Care Compunetics, 3, 168-175.

12. Gribbin, P. J. (1989). Development of 360/370 architecture - A plain man's view. Retrieved from http://www.leeandmelindavarian.com/Melinda/gribbin.pdf. 
13. Harbin, T. \& Baum, N. (2014). The vital two minutes of the patient encounter. The Journal of Medical Practice Management: MPM, 29(4), 248-249. Retrieved from https://search-proquestcom.contentproxy.phoenix.edu/docview/1524878168?accountid=134 061

14. Hoeren, T., \& Niehoff, M. (2018). Artificial intelligence in medical diagnoses and the right to explanation. European Data Protection Law Review (EDPL), 4(3), 308-319. doi:10.21552/edpl/2018/3/9

15. Hsueh, S. (2012). A fuzzy utility-based multi-criteria model for evaluating households' energy conservation performance: A Taiwanese case study. Energies, 5(8), 2818-2834. doi:10.3390/en5082818

16. Kilman, D. G., \& Forslund, D. W. (1997). An international collaboratory based on virtual patient records. Communications of the ACM, 40(8), 111-117. doi:10.1145/257874.257898

17. Loo, R. (2002). The delphi method: A powerful tool for strategic $\begin{array}{lll}\text { management. } & \text { 76licing, }\end{array}$ doi:10.1108/13639510210450677

18. Loveland, S., Dow, E. M., LeFevre, F., Beyer, D., \& Chan, P. F. (2008). Leveraging virtualization to optimize high-availability system configurations. IBM Systems Journal, 47(4), 591-604. doi:10.1147/SJ.2008.5386515

19. Marco, A. P. (2004). The virtual patient encounter - units of service in the electronic age. Physician Executive, 30(3), 32-34. Retrieved from https://search-proquestcom.contentproxy.phoenix.edu/docview/200057620?accountid=1340 61

20. Microsoft. (2019). Hyper-V server. Retrieved from https://www.microsoft.com/en-us/cloud-platform/servervirtualization.

21. Miseviciene, R., Ambraziene, D., Tuminauskas, R., \& Pazereckas, N. (2012). Educational infrastructure using virtualization technologies: Experience at Kaunas University of Technology. Informatics in Education, 11(2). Retrieved from https://search-proquestcom.contentproxy.phoenix.edu/docview/1112240702?accountid=134 061

22. Munro, J. (2001). Virtual machines \& VMware, part I. Retrieved from http://atlas.binus.ac.id/PDC/EasWeb2.nsf/0/d6f02e9dba8b088247256 bf7001f3bd3/\$FILE/Virtual\%20Machines\%20_VMware.pdf.

23. Parente, S. T., \& McCullough, J. S. (2009). Health information technology and patient safety: Evidence from panel data. Health Affairs, 28(2), 357-360. doi:10.1377/hlthaff.28.2.357 
24. Park, Y. R. \& Sharma, S. (2009). Providing service using a virtualization infrastructure. Clute Institute for Academic Research, 2(2), 17-22. Retrieved from https://search-proquestcom.contentproxy.phoenix.edu/docview/195627357?accountid=1340 61

25. Porter, M. (2008). The five competitive forces that shape strategy. Harvard Business Review. 25-40.

26. Purcarea, V. L., Petrescu, D. G., Gheorghe, I. R., \& Petrescu, C. M. (2011). Optimizing the technological and informational relationship of the health care process and of the communication between physician and patient: Factors that have an impact on the process of diagnosis from the physician's and the patient's perspectives. Journal of Medicine and Life, 4(2), 198-206. Retrieved from https://search-proquestcom.contentproxy.phoenix.edu/docview/876966298?accountid=1340 61

27. QSR International. (2018). NVivo ${ }^{\circledR}$. Retrieved from https://www.qsrinternational.com/nvivo/home.

28. Reiner, B. I. (2011, April). Improving healthcare delivery through patient informatics and quality centric data. Journal of Digital Imaging, 24, 177-178. doi:10.1007/s10278-011-9363-4

29. Robb, R. (2008). Medical imaging and virtual reality: A personal perspective. Virtual Reality, 12, 235-257. doi:10.1007/s10055-0080104-z

30. Schimke, I. (2009). Quality and timeliness in medical laboratory testing. Analytical \& Bioanalytical Chemistry, 393(5), 1499-1504. doi:10.1007/s00216-008-2349-5

31. Shapiro, J. (1993). The use of narrative in the doctor-patient encounter. Family Systems Medicine, 11(1), 47-53. doi:10.1037/h0089128

32. Spink, T., Wagstaff, H., \& Franke, B. (2016). Hardware-accelerated cross-architecture full-system virtualization. ACM Transactions on Architecture and Code Optimization (TACO), 13(4), 36. doi:10.1145/2996798

33. SurveyMonkey® . (2018). $\quad$ Retrieved from https://www.surveymonkey.com/.

34. Takakuma, K. M., Shofer, F. S., Boedec, C., \& Reyes, I. M. (2003). The effect of bedside registration on patient encounter times in an urban academic emergency department. Academic Emergency Medicine, 10(5), 525.

35. Van Vleck, T. (2013). The IBM 360/67 and CP/CMS. Retrieved from http://www.cedix.de/VorlesMirror/Band2/multicians.pdf. 
36. VMware.

(2019).

ESXi.

Retrieved

from http://www.vmware.com/products/vsphere/esxi-andesx/overview.html.

37. White, R. E. (2008). Health information technology will shift the medical care paradigm. Journal of General Internal Medicine, 23(4), 495-499. doi:10.1007/s11606-007-0394-y

38. Yaffe, M. J. (2019). Emergence of "big data" and its potential and current limitations in medical imaging. Seminars in Nuclear Medicine, 49, 94-104. doi:10.1053/j.semnuclmed.2018.11.010

39. Yehuda, B. (1999). Response rate in academic studies - a comparative analysis. Human Relations, 52(4), 421-438. doi:10.1177/001872679905200401

40. Young, A. S., Mintz, J., Cohen, A. N., \& Chinman, M. J. (2004). A network-based system to improve care for schizophrenia: The medical informatics network tool (MINT). Journal of the American Medical Informatics Association, 11(5), 358-367. Retrieved from https://search-proquest-

com.contentproxy.phoenix.edu/docview/220780938?accountid=1340 61 